\title{
ANGIOSPERM PHYLOGENY
}

\section{Flowering Plant Systematics}

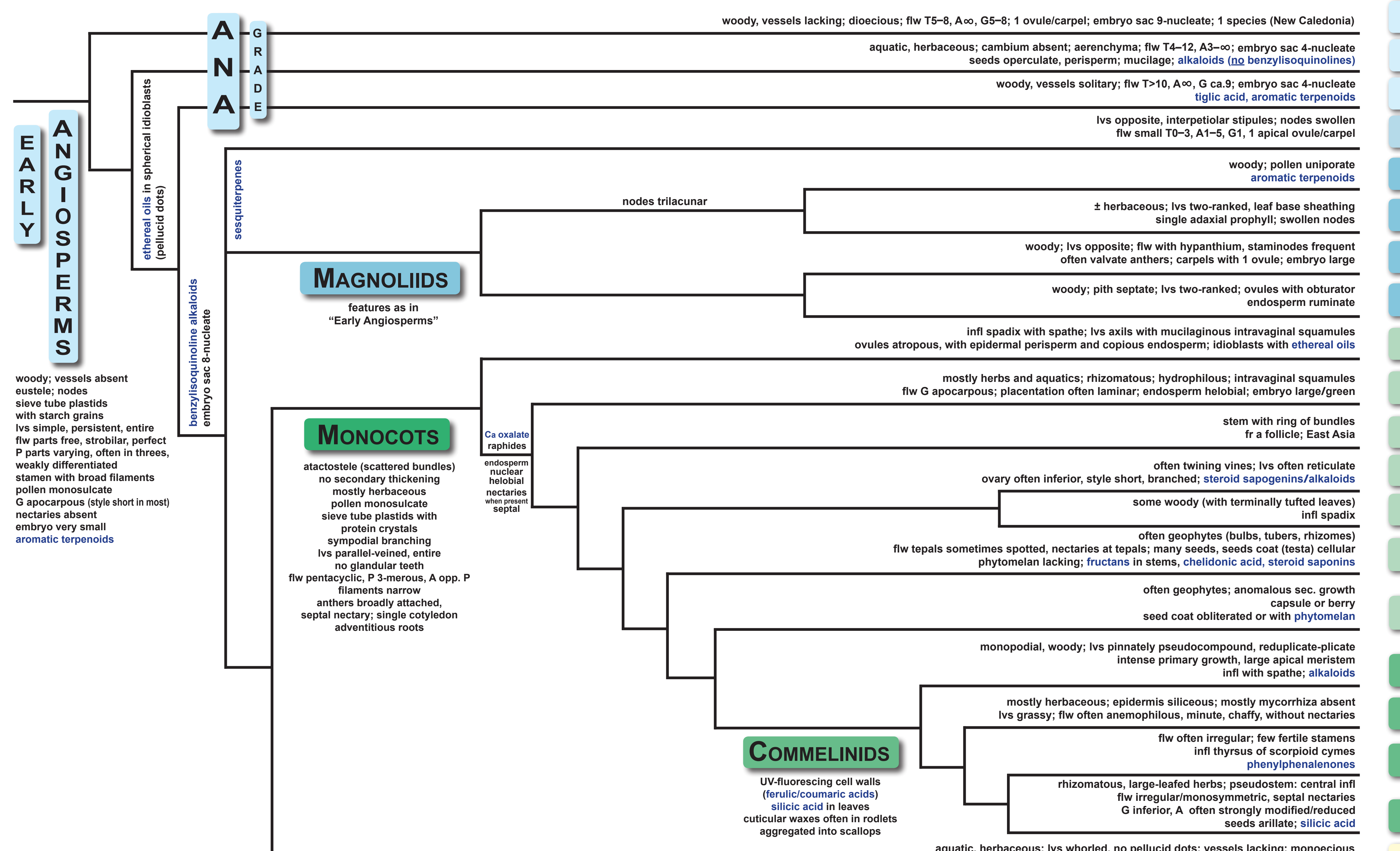

AMBORELLALES Amborellaceae

NYMPHAEALES Cabombaceae Hydatellaceae Nymphaeaceae

AUSTROBAILEYALES Austrobaileyaceae Schisandraceae (incl. llliciaceae) Trimeniaceae Chloranthales Chloranthaceae

Canellales Canellaceae Winteraceae

\begin{tabular}{l|lll} 
PIPERALES Aristolochiaceae (incl. Hydnoraceae) Piperaceae Saururaceae &
\end{tabular}

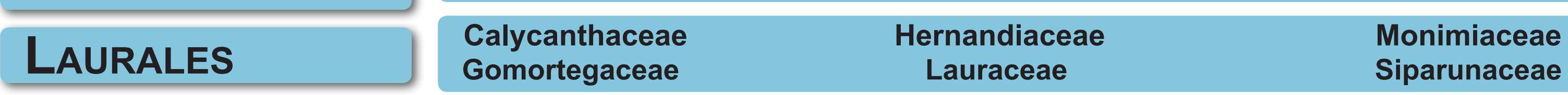

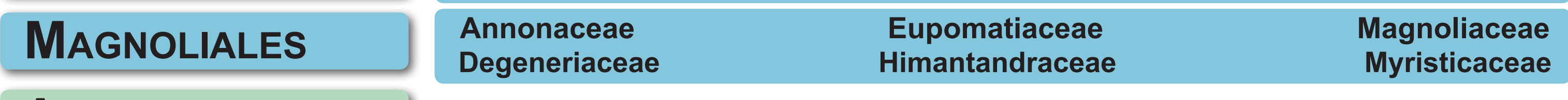

Acorales Acoraceae

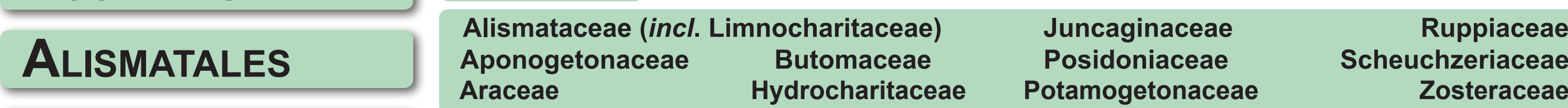

Petrosaviales Petrosaviaceae

DIOSCOREALES Burmanniaceae Dioscoreaceae Nartheciaceae Taccaceae

PANDANALES Cyclanthaceae Pandanaceae Velloziaceae

\begin{tabular}{l|llll} 
LILIALES & $\begin{array}{l}\text { Alstroemeriaceae } \\
\text { Colchicaceae }\end{array}$ & $\begin{array}{c}\text { Corsiaceae } \\
\text { Liliaceae }\end{array}$ & $\begin{array}{c}\text { Melanthiaceae } \\
\text { Petermanniaceae }\end{array}$ & $\begin{array}{c}\text { Philesiaceae } \\
\text { Smilacaceae }\end{array}$ \\
\hline
\end{tabular}

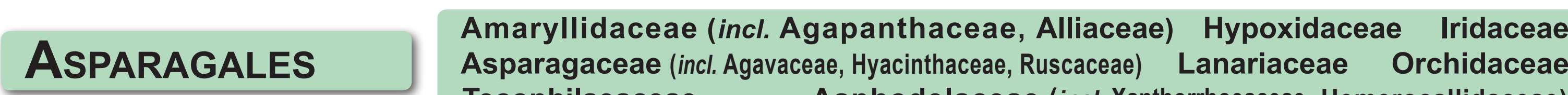

ARECALES Tecophilaeaceae Asphodelaceae (incl. Xanthorrhoeaceae, Hemerocallidaceae)

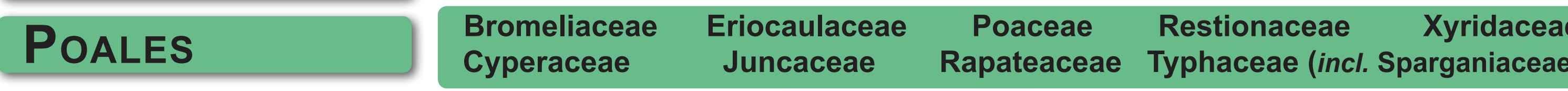

COMMELINALES Commelinaceae Haemodoraceae Pontederiaceae

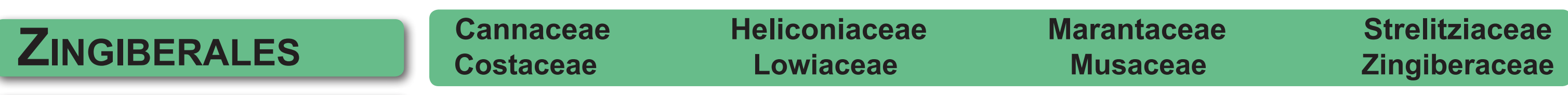

Ceratophyllales] Ceratophyllaceae

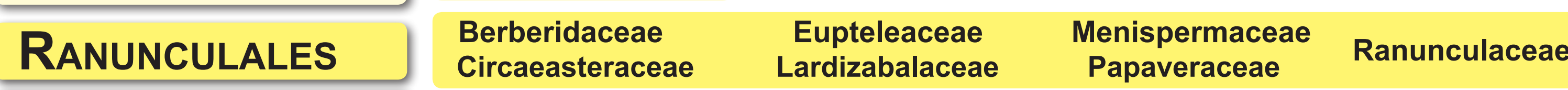

PROTEALES Nelumbonaceae Platanaceae Proteaceae Sabiaceae

TROCHODENDRALES Trochodendraceae

BUXALES Buxaceae (incl. Haptanthaceae)

GUNNERALES Gunneraceae Myrothamnaceae

Dilleniales Dilleniaceae

SAXIFRAGALES $\begin{aligned} & \text { Altingiaceae } \\ & \text { Cercidiphyllaceae }\end{aligned} \begin{gathered}\text { Cynomoriaceae } \\ \text { Grossulariaceae }\end{gathered} \begin{gathered}\text { Daphniphllaceae } \\ \text { Hamamelidaceae } \\ \text { Paeoniaceae }\end{gathered}$

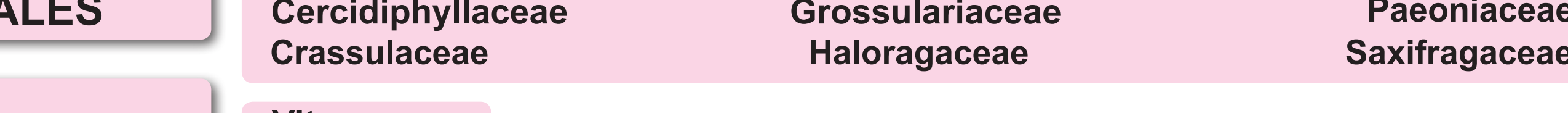

ZYGOPHYLLALES Krameriaceae Zygophyllaceae

CELASTRALES Celastraceae (incl. Hippocrateaceae, Brexiaceae, Parnassiaceae) Lepidobotryaceae

\begin{tabular}{l|llll} 
OXALIDALES & $\begin{array}{l}\text { Brunelliaceae } \\
\text { Cephalotaceae }\end{array}$ & $\begin{array}{l}\text { Connaraceae } \\
\text { Cunoniaceae }\end{array}$ & $\begin{array}{c}\text { Elaeocarpaceae } \\
\text { Huaceae }\end{array}$ & Oxalidaceae \\
\hline
\end{tabular}

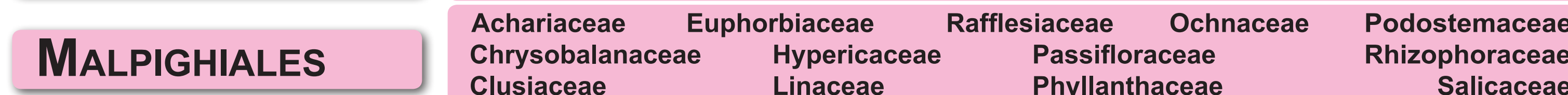

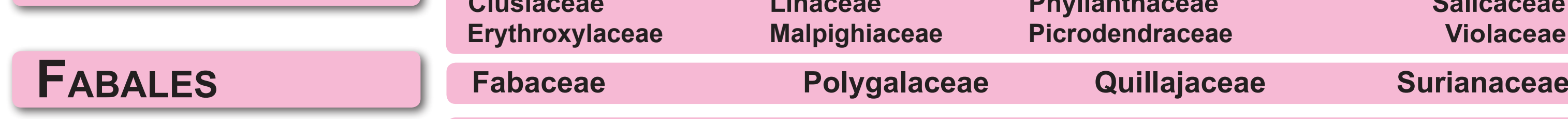

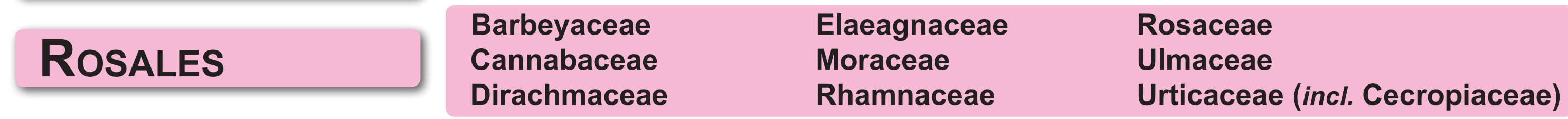

\begin{tabular}{l|llll} 
CUCURBITALES & $\begin{array}{l}\text { Apodanthaceae } \\
\text { Anisophyllaceae }\end{array}$ & $\begin{array}{l}\text { Begoniaceae } \\
\text { Coriariaceae }\end{array}$ & $\begin{array}{c}\text { Corynocarpaceae } \\
\text { Cucurbitaceae }\end{array}$ & $\begin{array}{r}\text { Datiscaceae } \\
\text { Tetramelaceae }\end{array}$ \\
\hline
\end{tabular}

\begin{tabular}{l|llll} 
FAGALES & $\begin{array}{l}\text { Betulaceae } \\
\text { Casuarinaceae }\end{array}$ & $\begin{array}{c}\text { Fagaceae } \\
\text { Juglandaceae }\end{array}$ & $\begin{array}{c}\text { Myricaceae } \\
\text { Nothofagaceae }\end{array}$ & $\begin{array}{c}\text { Rhoipteleaceae } \\
\text { Ticodendraceae }\end{array}$ \\
\hline
\end{tabular}

GERANIALES Geraniaceae Francoaceae (incl. Ledocarpaceae, Melianthaceae, Vivianiaceae)

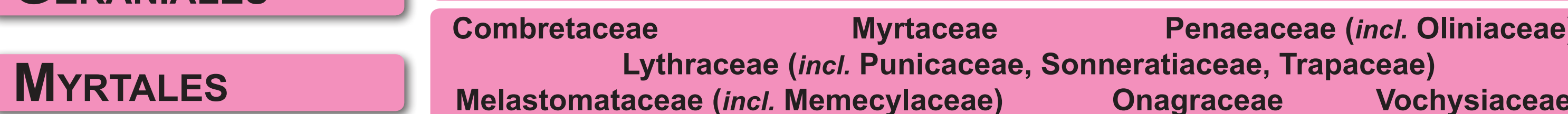

\begin{tabular}{l|lll}
\hline CROSSOSOMATALES & $\begin{array}{l}\text { Crossosomataceae } \\
\text { Geissolomataceae }\end{array}$ & $\begin{array}{l}\text { Stachyuraceae } \\
\text { Staphyleaceae }\end{array}$ & Strasburgeriaceae \\
\hline PICRAMIALS & &
\end{tabular}

PICRAMNIALES Picramniaceae

\begin{tabular}{|l|lll} 
SAPINDALES & $\begin{array}{l}\text { Anacardiaceae } \\
\text { Biebersteiniaceae }\end{array}$ & $\begin{array}{c}\text { Burseraceae } \\
\text { Meliaceae }\end{array}$ & $\begin{array}{l}\text { Kirkiaceae } \\
\text { Rutaceae }\end{array}$ \\
\hline Nitraceae & $\begin{array}{r}\text { Sapindaceae } \\
\text { Simaroubaceae }\end{array}$
\end{tabular}

\begin{tabular}{l|lll}
\hline HUERTEALES Dipentodontaceae Gerrardinaceae Petenaeaceae Tapisciaceae &
\end{tabular}

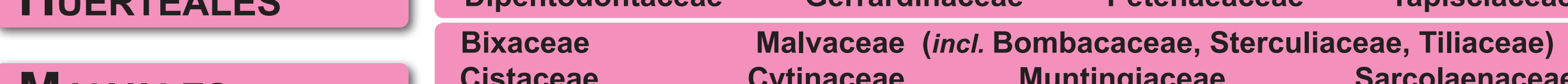

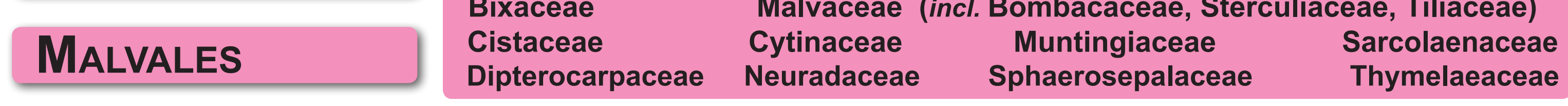

\begin{tabular}{|l|lllr}
\hline BRASSICALES & $\begin{array}{l}\text { Bataceae } \\
\text { Brassicaceae } \\
\text { Capparaceae }\end{array}$ & $\begin{array}{l}\text { Caricaceae } \\
\text { Cleomaceae } \\
\text { Koeberliniaceae }\end{array}$ & $\begin{array}{l}\text { Limnanthaceae } \\
\text { Moringaceae } \\
\text { Resedaceae }\end{array}$ & $\begin{array}{r}\text { Salvadoraceae } \\
\text { Tovariaceae } \\
\text { Tropaeolaceae }\end{array}$ \\
\hline
\end{tabular}

BERBERIDOPSIDALES Aextoxicaceae Berberidopsidaceae

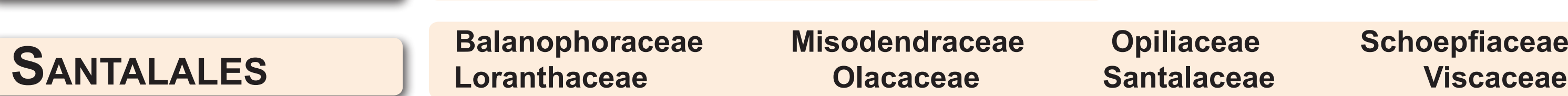

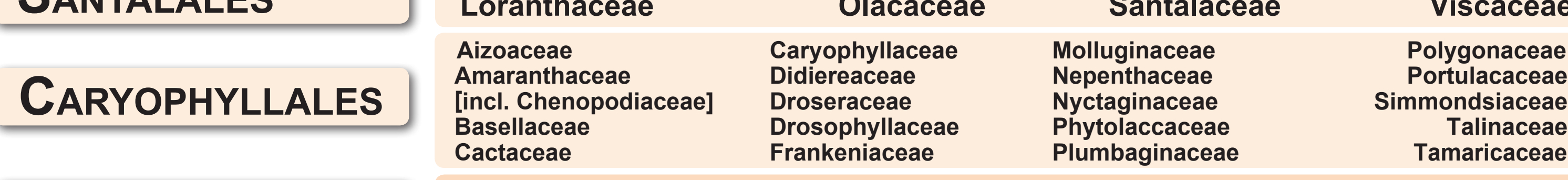

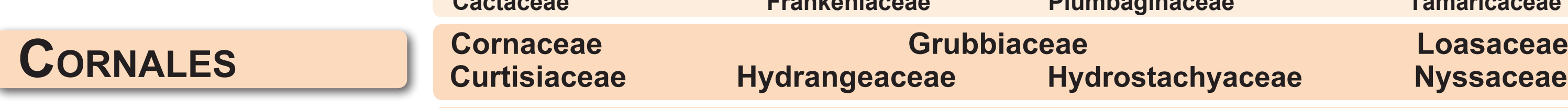

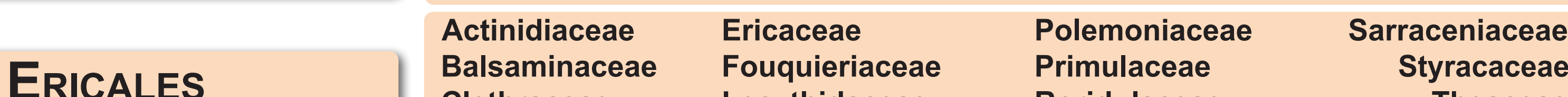

$\begin{array}{lll}\text { Clethraceae } & \text { Lecythidaceae } & \begin{array}{l}\text { Roridulaceae } \\ \text { Sapotaceae }\end{array}\end{array} \begin{array}{r}\text { Theaceae } \\ \text { Myrsinaceae }\end{array}$

ICACINALES Oncothecaceae Icacinaceae

MetTeniusales Metteniusaceae

GARRYALES Eucommiaceae Garryaceae (incl. Aucubaceae)

Theodor C. H. Cole, Dipl. Biol.
Institute of Pharmacy and Molecular Biotechnology (IPMB) Heidelberg University
Im Neuenheimer Feld $364, \mathrm{D}-69120$ Heidelberg, Germany

Freie Universität

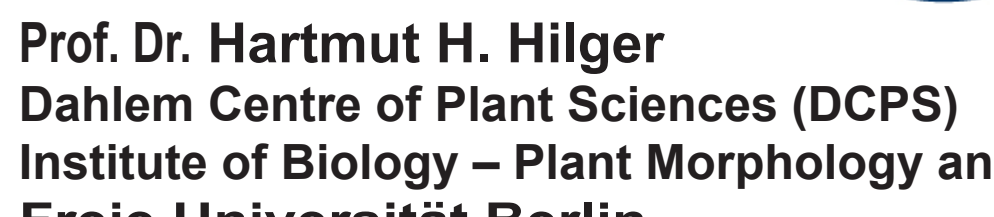

Freie Universitiat Berlin
Attensteinstr. 6 , D-14195 Berin, Germany

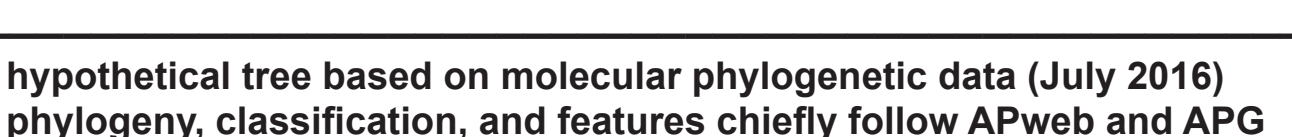

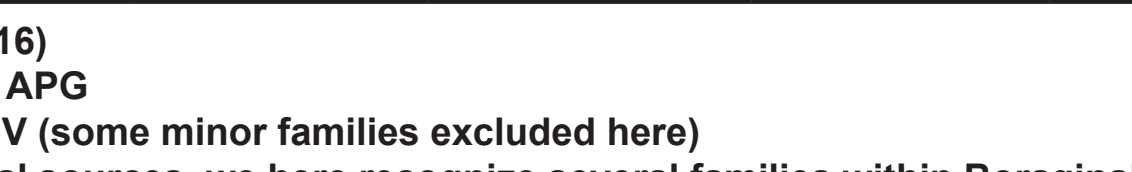

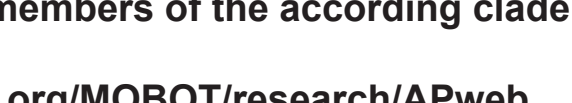

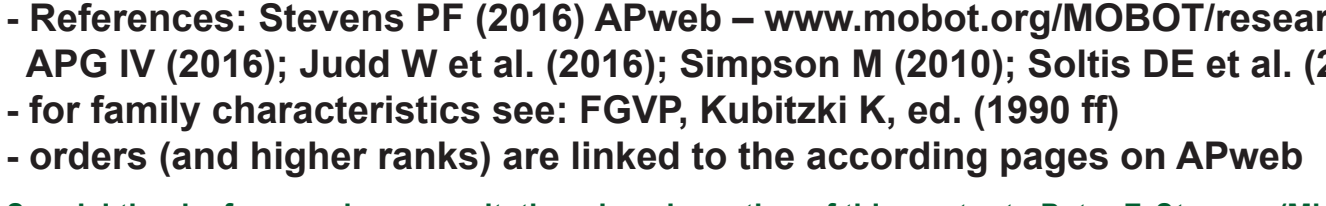

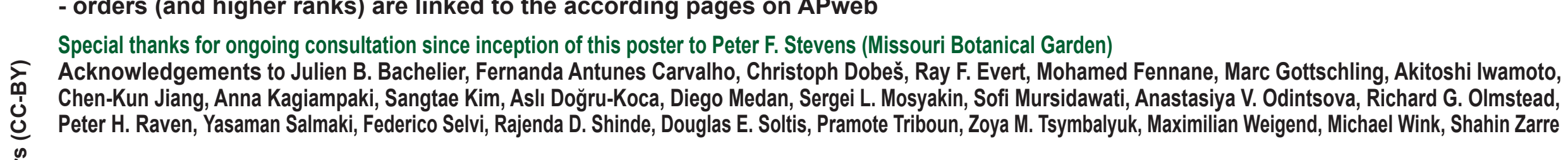

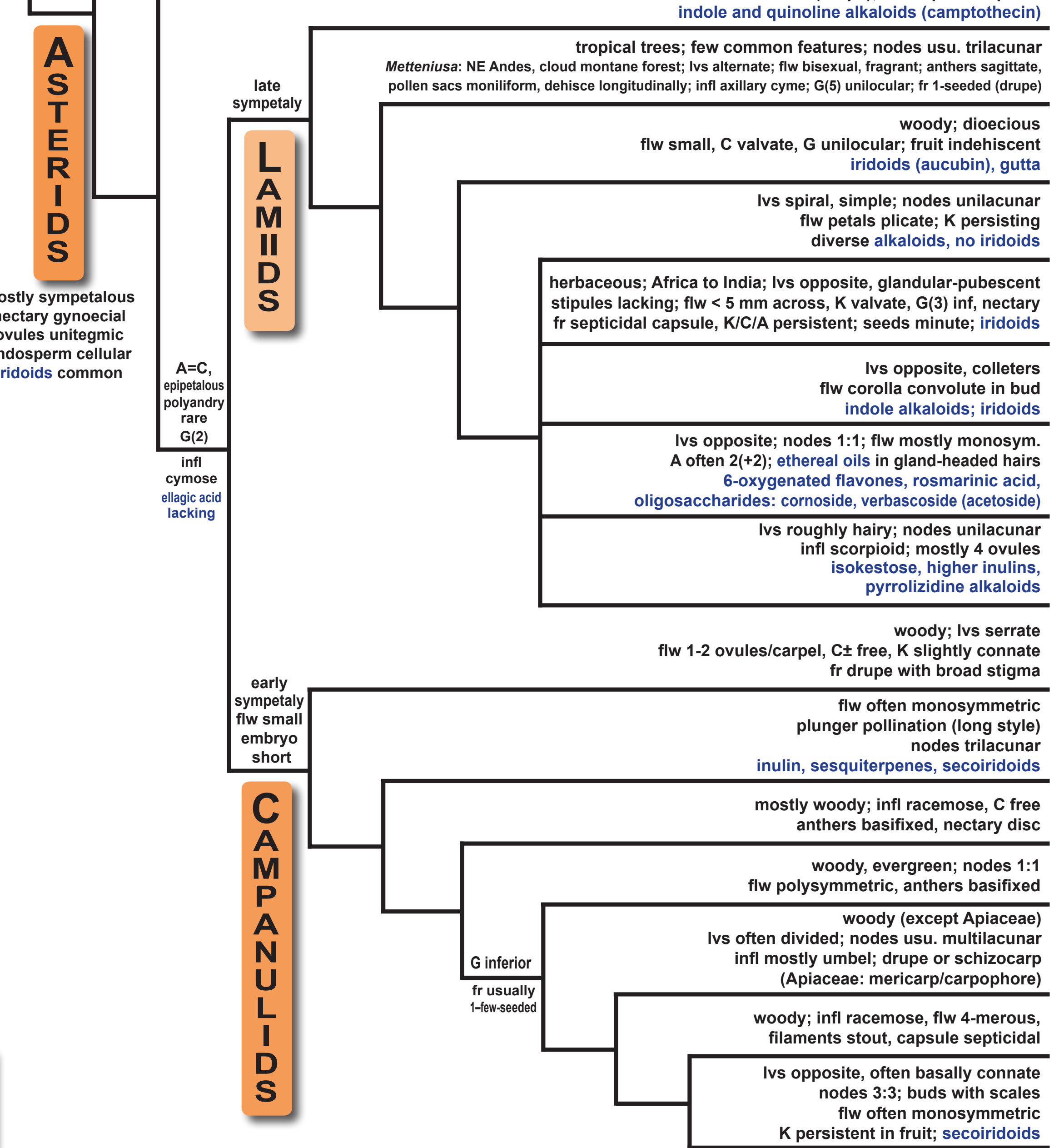

SOLANALES Convolvulaceae (incl. Cuscutaceae) Solanaceae (incl. Nolanaceae)

VAHLIALES Vahliaceae

GENTIANALES $\begin{gathered}\text { Apocynaceae (incl. Asclepiadaceae) } \\ \text { Gentianaceae } \\ \text { Gelsemiaceae }\end{gathered} \quad \begin{gathered}\text { Loganiaceae } \\ \text { Rubiaceae }\end{gathered}$

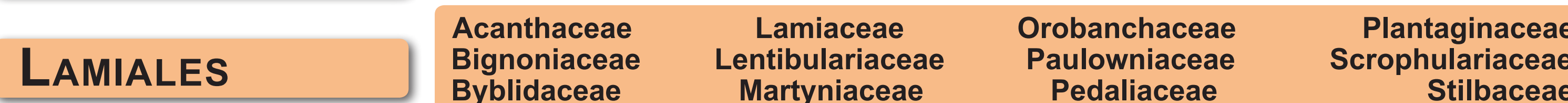

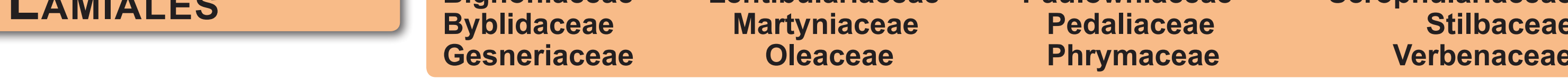

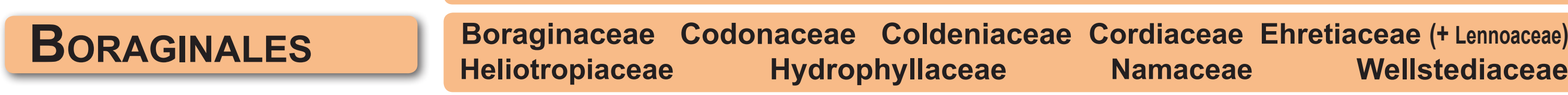

AQUIFOLIALES Aquifoliaceae Cardiopteridaceae Stemonuraceae

\begin{tabular}{llll} 
ASTERALES & $\begin{array}{l}\text { Asteraceae } \\
\text { Calycraceae }\end{array}$ & $\begin{array}{c}\text { Goodeniaceae } \\
\text { Menvanthaceae }\end{array}$ & $\begin{array}{c}\text { Pentaphragmataceae } \\
\text { Rousseacaeae }\end{array}$ \\
\hline
\end{tabular}

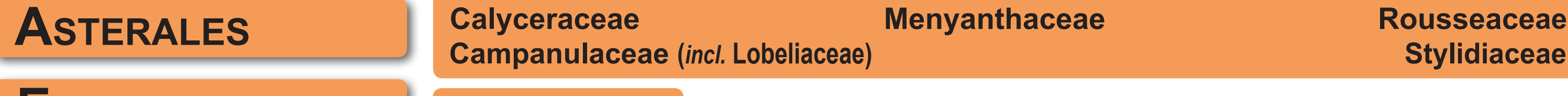

Escalloniales Escalloniaceae

BRUNIALES Bruniaceae Columelliaceae (incl. Desfontainia)

\begin{tabular}{l|lll}
\hline APIALES & $\begin{array}{c}\text { Apiaceae } \\
\text { Araliaceae }\end{array}$ & $\begin{array}{c}\text { Griseliniaceae } \\
\text { Myyodocarpaceae }\end{array}$ & $\begin{array}{c}\text { Pennantiaceae } \\
\text { Pittosporaceae }\end{array}$ \\
\hline
\end{tabular}

PARACRYPHIALES Paracryphiaceae

DIPSACALES Adoxaceae 